\title{
Interoperator fixed-mobile network sharing
}

\author{
Ireneusz Szcześniak, Piotr Chołda, Andrzej R. Pach \\ AGH University of Science and Technology \\ Department of Telecommunications \\ al. Mickiewicza 30 \\ 30-059 Krakow \\ Poland
}

\author{
Bożena Woźna-Szcześniak \\ Institute of Mathematics and Computer Science \\ Jan Długosz University \\ al. Armii Krajowej 13/15 \\ 42-200 Częstochowa \\ Poland
}

\begin{abstract}
We propose the novel idea of interoperator fixedmobile network sharing, which can be software-defined and readily-deployed. We study the benefits which the sharing brings in terms of resiliency, and show that, with the appropriate placement of a few active nodes, the mean service downtime can be reduced more than threefold by providing interoperator communication to as little as one optical network unit in one hundred. The implementation of the proposed idea can be carried out in stages when needed (the pay-as-you-grow deployment), and in those parts of the network where high service availability is needed most, e.g., in a business district. While the performance should expectedly increase, we show the resiliency is brought almost out of thin air by using redundant resources of different operators. We evaluated the service availability for 87400 networks with the relative standard error of the sample mean below $1 \%$.
\end{abstract}

Index Terms-interoperator network sharing, fixed-mobile network, passive optical network, backhaul, availability, resiliency

\section{INTRODUCTION}

A fixed-mobile network (FMN) delivers services for fixed users (FUs) to their premises, and for mobile users (MUs) to their user equipment (UE) such as mobile phones or mobile routers. This fixed-mobile convergence allows a network operator to consolidate and simplify business. A FMN is composed of the radio access network (RAN), e.g., the Long-Term Evolution (LTE) network, and the backhaul, e.g., a passive optical network (PON). The backhaul connects both FUs and the RAN base stations (BSs).

Maintaining and upgrading a FMN is expensive, and the scarce radio spectrum for RANs is getting more expensive. For these reasons, the interoperator sharing of the network and spectrum is gaining prominence, because it offers to lower costs and increase revenue through better network and spectrum utilization [1]. But sharing can have various forms.

Currently network sharing between competing operators is a fact [2], but it is limited to the physical network infrastructure only (buildings, towers, etc.). Some operators merge their networks into a single network, and then own and use it together in a marriage-like fashion. These forms of sharing are of the legal, not technological nature.

We concentrate on the sharing enabled by technology, where an operator is able to temporarily rent resources from other operators. Unlike in network merging, an operator can use a resource without owning it. A resource can be anything used to implement a service: it can be a fixed or mobile resource, including spectrum. The traffic and technical difficulties which operators experience at a given time and place can differ substantially indeed between different operators, and the interoperator sharing would allow the operators to do better.

Sharing in FMNs pertains to RAN sharing and backhaul sharing. In RANs, the dynamic spectrum access (DSA) allows for various forms of spectrum sharing. Sharing of the backhaul could be realized with a virtual local area network (VLAN) or a Carrier Ethernet network. Software-definition augments the implementation.

The resiliency of the future FMN is crucial, but in the currently deployed FMNs it is missing. For instance, the currently deployed LTE is not resilient, and so are not the PONs. Resiliency is one of the key requirements of the fifth generation (5G) networks [3], and of the next generation PONs (NG-PONs) [4].

FMNs are being broadly researched and developed to deliver the required performance and resiliency [5]. Radio access technologies (RATs) have been proposed to use cognitivity, virtualization, coordinated multipoint transmission (CoMT), and more sophisticated modulation formats. The backhaul is evolving from the copper or microwave networks to passive optical networks (PONs), and even possibly to radio-over-fiber (RoF) networks [6]. PONs are currently being deployed as the backhaul, and the NG-PONs are being intensively researched for FMNs [7]. To the best of our knowledge, no interoperator FMN sharing has been proposed before.

Our contribution is the novel idea of interoperator FMN sharing, and the evaluation of the benefits the sharing brings in terms of resiliency. The benefits are mainly realized by the communication between different operators either wirelessly or optically. Beside the expected gain in performance, we argue that the resiliency is almost there: the currently-deployed FMNs of different operators have evolved independently and redundantly, and when they are shared, their redundancy can be used to implement resiliency.

The article is organized as follows. First, in the following Section [II we review key related works, and in Section III we describe the proposed interoperator FMN sharing. Next, in Section [V] we describe the evaluation setting, and in Section VI we report on the obtained numerical results. Finally, Section $[\mathrm{VII}$ concludes the article. 


\section{RELATED WORKS}

Mobile network sharing has long been used, allowing for roaming or virtual mobile network operators to exist, where a mobile operator accepts traffic directly from the users of a different operator. In [2] the authors study the virtualization support for this traditional sharing. In [8] the authors discuss novel FMN architectures. The hallmark of our proposed sharing is the interoperator communication, where traffic is exchanged by different operators between their access networks.

In [9], the authors propose a number of wireless protection methods for FMNs. There a single network is considered, without sharing it with a different operator. Wireless access points connected to a PON are allowed to offer backup connectivity to those wireless access points which lost the PON connectivity. These methods do not protect against, for example, the failure of the feeder fiber, while our method does.

The various forms of DSA have been widely embraced by researchers, industry and legislatures, and are regarded as the key enablers of 5G. DSA is being legislated worldwide, and a number of standardization bodies are working on it [10].

The two most prominent types of DSA are the orthogonal spectrum sharing (OSS) and the non-orthogonal spectrum sharing (NSS) [11]. In OSS, the operators coordinate the shared bands (using, e.g., the X2 interface in LTE), so that a given band is used exclusively by a single operator at a given time and place. In NSS, a given band is used simultaneously by a number of operators at a given time and place. In NSS, spectrum sensing is key to learn of used and unused bands, and to minimize radio interference.

PONs are successful mainly because of the cost-effective tree topology. First, the feeder fiber starts at the optical line terminal (OLT) in the central office (CO), and ends at the first remote node $(\mathrm{RN})$ in some district. From there, the distribution fibers lead to further RNs in various neighborhoods, possibly through further RNs. Finally, the last-mile fibers deliver the service to customer premises.

NG-PONs should support direct communication between ONUs, without the OLT relaying the data, in order to support direct communication between BSs (connected to ONUs) required by future RANs. However, in legacy PONs, ONUs do not communicate directly with each other, but through the OLT. To this end, in [7] the authors propose two novel NG-PON architectures. Interestingly, the authors propose to cleverly use a circulator as a passive RN, which would allow for some limited communication between BSs without the OLT. Another solution is to use the active RNs, which would also enable NG-PONs to have larger splitting ratios and longer reach [12].

PONs are vulnerable to service disruption, because of the tree architecture. Failure of the OLT or the feeder fiber brings down the entire PON. Making a PON resilient is becoming more important, but requires expensive redundant infrastructure, fibers and hardware. In [13] the authors review PON resiliency mechanisms and propose their own mechanism for cost-effective resiliency on request.

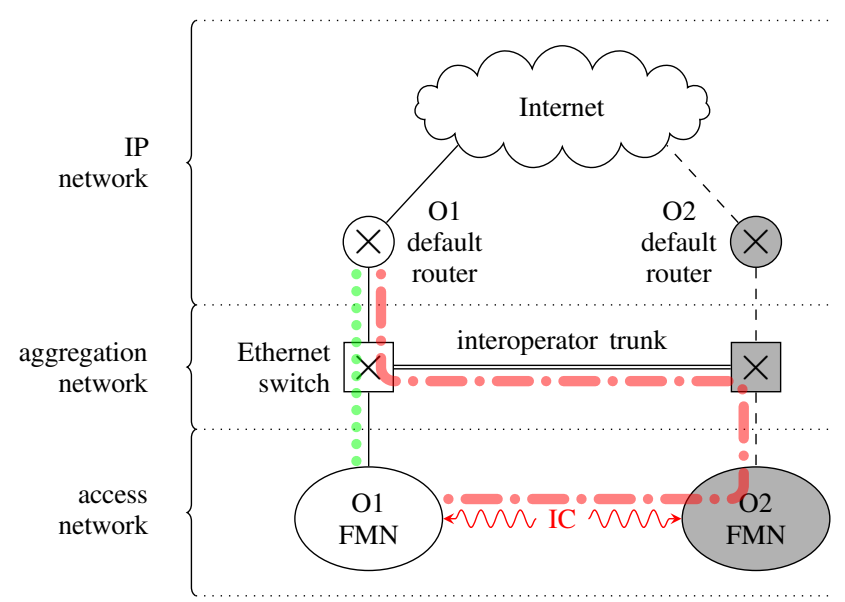

Fig. 1. General network architecture.

\section{INTEROPERATOR FMN SHARING}

There are two operators, Operator $1(\mathrm{O} 1)$ and Operator 2 (O2) who want to share their FMNs, including spectrum. An operator owns its FMN and spectrum independently of the other operator.

In the proposed sharing we introduce the interoperator communication (IC) to the access network. The traffic of $\mathrm{O} 1$ accepted by the access network of $\mathrm{O} 2$ is forwarded back to the network of $\mathrm{O} 1$ through the aggregation network. The IC makes the $\mathrm{O} 1$ service resilient to major failures of its access network (like a power outage at a $\mathrm{CO}$ ), which otherwise would bring the service down.

Figures 1 and 2 show the network architecture under study. For O1, nodes are filled white and links are drawn solid, and for $\mathrm{O} 2$, nodes are filled gray and links are drawn dashed.

The general network architecture is shown in Fig. 1, where $\mathrm{O} 1$ and $\mathrm{O} 2$ share their networks. To keep the example simple, the access network has only two FMNs, the aggregation network has only two Ethernet switches, and the Internet Protocol (IP) network has only two default routers. The thick dotted path shows the working path of the frames, and the thick dash-dotted path shows the backup path provided by the IC.

At the IP layer, MUs and FUs of the FMNs communicate with the default routers of their operators. No IP routing is carried out in the access or aggregation networks, since these networks switch Ethernet frames only, which is a valid assumption for aggregation and access networks.

In the FMNs, operators share their networks using the IC. In the aggregation network, operators share their networks using interoperator Ethernet trunks, which carry modified Ethernet frames. The Ethernet frames are modified, so that they can be sent over the Ethernet network of the other operator. Frames can be modified using stacked VLANs (Q-in-Q) or stacked MACs (Mac-in-Mac); both should do its job, but Mac-in-Mac would be better suited for large scale deployments.

We abstract the details of specific technologies and make core assumptions in order to take into account the currently 


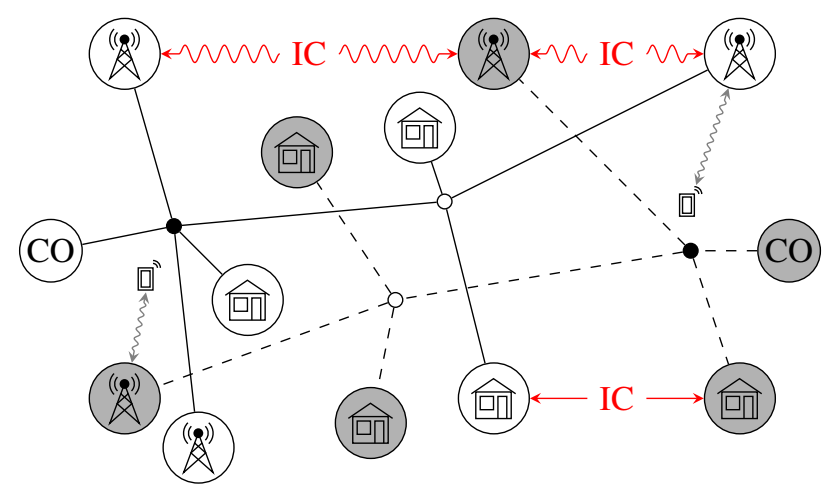

Fig. 2. Interoperator FMN sharing, where $\mathrm{CO}$ is a central office, IC is the interoperator communication, $\bullet$ is a passive remote node, $\circ$ is an active remote node, $\square^{\prime \prime}$ is a mobile user, ${ }^{\prime}$ is a base station, and 画 is a fixed user.

deployed networks (e.g., LTE, PONs) and the plausible future networks (e.g., LTE-A, NG-PON, RoF). We describe the assumption about the RAN first, and then about the backhaul.

As for the RANs, we assume that a BS is connected to the backhaul with a single fiber. A BS carries out the communication with MUs, and with the BSs of the other operator. The MU equipment is unaware of the IC which is taking place between BSs, and so there is no need to modify the MU equipment. In the case of OSS, the BS software would have to be upgraded to enable our proposed sharing, but without the need to install new hardware, like the spectrum sensing hardware as would be required in the case of NSS.

As for the backhaul, we assume there is one point of connection of the backhaul to the central office (e.g., OLT in PONs). We assume the downstream and upstream throughput of the backhaul, e.g., $10 \mathrm{~Gb} / \mathrm{s}$ or $100 \mathrm{~Gb} / \mathrm{s}$, is shared between a large number (e.g., 1024) of clients (e.g., ONUs in PONs) which are either BSs or FUs. We assume the tree architecture of the optical distribution network (ODN).

We need active RNs to implement the proposed network sharing, because they are able to accomplish what passive RNs cannot: diverging upstream traffic to a detour downstream path if an upstream path fails. It is hard to argue for active RNs in PONs, because PONs are successful mainly because of its passive ODN with passive RNs, which are cheaper and more robust than active RNs. Nevertheless, we rely on active RNs, because we believe they will become more spread for two important reasons. First, active RNs can implement the direct communication between ONUs required by 5G. Second, active RNs (e.g., range extenders) are already used in PONs, and they are likely to be more popular with NG-PONs, which can have a large splitting ratio (e.g., 1:32) and be long-reach (above 100 $\mathrm{km})$.

Fig. 2 shows the IC between two FMNs. Each of the operators has a $\mathrm{CO}$ at which an ODN of the tree topology is rooted. FUs and BSs are connected to ODNs, and MUs communicate with BSs. The IC is taking place wirelessly between BSs of different operators and optically between fixed users of different operators.

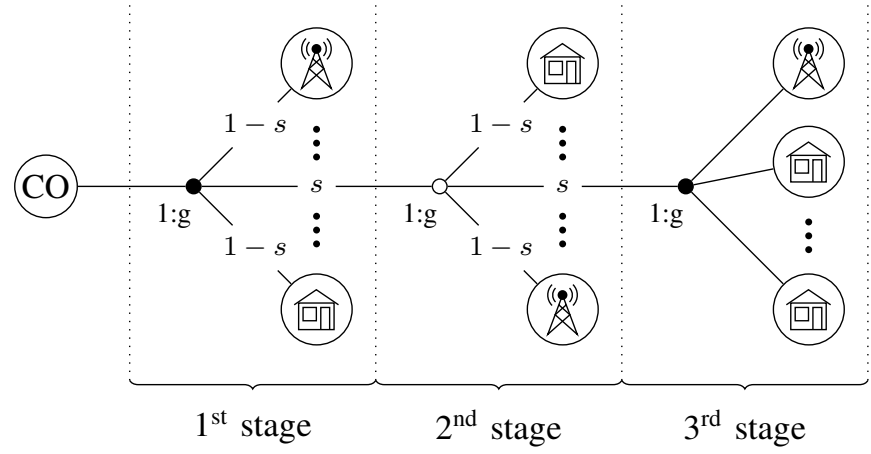

Fig. 3. PON topology model, where $\mathrm{CO}$ is a central office, $\bullet$ is a passive remote node, $\circ$ is an active remote node, is a base station, and 畐 is a fixed user.

\section{EVALUATION SCENARIOS}

We consider two evaluation scenarios. They both are very similar and differ only in the way the RN type is chosen.

\section{A. First scenario}

Fig. 3 illustrates the first scenario. The PON has the depth of three stages. The PON can have many second and third stages, but in the figure we show only one of each. In the first stage a passive $\mathrm{RN}$ with the $1: \mathrm{g}$ splitting ratio is used. The probability that a fiber coming out of the RN goes to a second stage is $s$ and, conversely, $(1-s)$ that it goes to an ONU, a FU or a BS.

The high 1:g splitting ratio and possibly long feeder and distribution fibers may require an active $\mathrm{RN}$, and so at the second stage we install an active RN. At the second stage the probabilities of $s$ and $(1-s)$ have the same meanings. Finally, at the third stage there is a passive RN installed, and all fibers reach an ONU. The last-mile fibers are typically short, and even with a 1:g or higher splitting ratio, passive RNs suffice.

An ONU is capable of the IC with probability $r$. These interoperator-communicating ONUs (IC-ONUs) can offer the Internet communication in the same way as the OLT does, while the remaining ONUs are non interoperatorcommunicating ONUs (NIC-ONUs).

For this PON topology of depth three, the given $s$, and the given $1: \mathrm{g}$ splitting ratio, the mean number $N$ of ONUs is given by (1).

$$
N=g(1-s+g s(1-s+g s))
$$

We assume $s=0.3$ and $g=32$, and so the number of ONUs is $N \approx 3187$, which is reasonable for NG-PONs. For instance, currently the XG-PON supports 1024 ONUs.

The availability values of the PON components are taken from [13], and they are summarized in Table \. The reported availability value for the passive $\mathrm{RN}$ is that for the 1:32 power splitter, and for the active RN is that for the OLT. The values reported for the feeder fiber, the distribution fiber and the lastmile fiber are calculated with the reported fiber availability per 
TABLE I

AVAilability VALUES.

\begin{tabular}{|r|l|}
\hline \multicolumn{1}{|c|}{ Component } & Availability \\
\hline OLT & 0.9999485 \\
ONU & 0.9999645 \\
passive remote node & 0.9999987 \\
active remote node & 0.9999485 \\
fiber per km & 0.9999429 \\
feeder fiber & 0.999429 \\
distribution fiber & 0.999829 \\
last-mile fiber & 0.99996 \\
\hline
\end{tabular}

$\mathrm{km}$ assuming that their mean lengths are $10 \mathrm{~km}, 3 \mathrm{~km}$ and 0.7 $\mathrm{km}$, respectively.

\section{B. Second scenario}

The second scenario differs from the first scenario only in the selection of the RN types, i.e., whether they are passive or active. While in the first scenario the type of a $\mathrm{RN}$ is given up front, in the second scenario it is given probabilistically: a RN is active with probability $q$. The second scenario allows us to study how the ONU service availability changes as a function of probability $q$.

\section{SERVice AVAILABILITy CALCUlation}

We want to calculate the mean ONU service availability (SA) for a given network taken from the network populations of the two scenarios. For the given network, we are provided the topology, the type of RNs, and the information on which ONUs are capable of the IC. The mean ONU SA is the arithmetic mean of the SAs of all ONUs (all IC-ONUs and NIC-ONUs). The SA of the IC-ONU equals the availability of the OLT, because it can rely on the IC. The problem is to calculate the SA of a given NIC-ONU.

The SA calculation for the proposed sharing is more difficult than for traditional PONs. The ONU SA for traditional PONs is calculated by following upstream a single path from the ONU to the OLT, and just multiplying the availabilities of the encountered components. In the proposed sharing, the availability calculation is more complicated for three reasons. First, in addition to the path from the NIC-ONU to the OLT, we need to consider the paths from the NIC-ONU to all the ICONUs. Second, the considered paths are not always upstream only: a path can be upstream-downstream at the same time, i.e., it can go upstream first and downstream next to reach an IC-ONU. Third, an upstream-downstream path can traverse some nodes and fibers twice, and their availabilities should be taken into account only once.

The SA of the given NIC-ONU is calculated by evaluating a reliability block diagram (RBD) of the service paths from the NIC-ONU to the OLT and all the IC-ONUs. Since a PON has the tree topology, the corresponding RBDs have the parallel and serial configurations only, without the crossover configurations, making the evaluation easy to implement programmatically with the recursive depth-first search. Nonetheless, the evaluation has some important intricacies, and we discuss them further below.
The recursive function $f(c, p)$ calculates the SA for the current node $c$, provided the previous node is $p$. Node $p$ preceded the current node $c$, i.e., node $p$ was the current node in the previous call of the function. The function is initially called with the NIC-ONU of interest as the current node, and with $p=$ null. The function recursively calls itself to calculate the availabilities of the RNs, and eventually of the OLT and other ONUs.

Function $f(c, p)$ is given by (2), where $a_{c}$ is the availability of node $c, u_{c}$ is the node upstream of node $c, u_{c} \rightarrow c$ is the upstream fiber of node $c, a_{u_{c} \rightarrow c}$ is the availability of that fiber, and $N_{c}$ is the set of neighbor nodes of node $c$. Symbols $V_{c}$, $h_{c}$, and $d_{c, v}$ are defined further down.

$$
f(c, p)=\left\{\begin{array}{cc}
a_{c} a_{u_{c} \rightarrow c} f\left(u_{c}, c\right) & 1^{\text {st }} \text { case } \\
0 & 2^{\text {nd }} \text { case } \\
a_{c} & 3^{\text {rd }} \text { case } \\
a_{c}\left(1-\prod_{\substack{i \in N_{c} \\
i \neq p}}\left(1-a_{i \rightarrow c} f(i, c)\right)\right) & 4^{\text {th }} \text { case } \\
h_{c}\left(1-\prod_{v \in V_{c}}\left(1-d_{c, v}\right)\right) & 5^{\text {th }} \text { case }
\end{array}\right.
$$

The cases of the function are as follows:

$1^{\text {st }}$ case is for the initial call of the function, i.e., when $c$ is an NIC-ONU and $p=$ null, which allows the function to reach the upstream node $u_{c}$,

$2^{\text {nd }}$ case applies when the function reaches an NIC-ONU from some previous node, i.e., $p \neq$ null, in which case no service is offered,

$3^{\text {rd }}$ case applies when $c$ is the OLT or an IC-ONU, which offer the service,

$4^{\text {th }}$ case applies when $c$ is an active $\mathrm{RN}$ or a passive $\mathrm{RN}$ reached from an upstream node, i.e., $p=u_{c}$, which offers to reach in parallel the neighbor nodes $N_{c}$ of node $c$, excluding node $p$,

$5^{\text {th }}$ case applies when $c$ is a passive $\mathrm{RN}$ reached from a downstream node, i.e., $p \neq u_{c}$, which is the most difficult case discussed below.

In the $5^{\text {th }}$ case the upstream-downstream paths exist for an NIC-ONU connected to a passive RN, when to the same passive segment (i.e., a sequence of passive RNs) there are ICONUs connected. The NIC-ONU can get service either from nodes reachable through the first active upstream node (the OLT or an active RN), or from an IC-ONU connected to the same passive segment. All paths for the NIC-ONU have the same shared path, starting at the first passive upstream RN for which the function was called, through the upstream fibers and possibly further passive upstream RNs, up to and including the first upstream active node. The availability of the shared path for node $c$ is $h_{c}$, and it has to be accounted for only once. From this shared path all parallel non-shared paths $V_{c}$ fork, i.e., the paths for service nodes reachable from the first active 


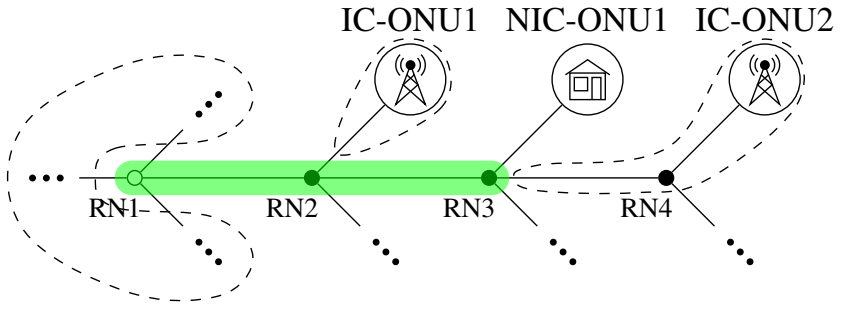

Fig. 4. Example for the $5^{\text {th }}$ case of function $f(c, p)$, where the thick line segment shows the shared path, the dashed curves show the non-shared paths, - is a passive remote node, and $\circ$ is an active remote node.

node, and the paths for the IC-ONUs connected to the same passive segment. The availability of the parallel non-shared path $v$ for node $c$ is $d_{c, v}$.

Fig. 4 shows an example for the $5^{\text {th }}$ case of function $f(c, p)$. We calculate the SA of node NIC-ONU1, which can get service from nodes reachable through node $\mathrm{RN} 1$, or from nodes IC-ONU1 and IC-ONU2. The function is called for node NIC-ONU1 first, and for node RN3 next, i.e., $c=\mathrm{RN} 3$. The thick line segment highlights the shared path with availability $h_{c}$, and the dashed curves show the parallel non-shared paths $V_{c}$ with availabilities $d_{c, v}$.

\section{EVALUATION RESULTS}

We evaluated the ONU SA by randomly generating network samples for the network populations with the given characteristics for the two scenarios. Each network sample has one hundred networks. The sample mean of the ONU SA is the arithmetic mean of the ONU SAs calculated for the networks in the sample. We deem the sample means credibly estimate the population means since the relative standard errors of all sample means are below $1 \%$. For both scenarios there were 874 populations considered, and 87400 networks evaluated. The software is available at [14].

\section{A. First scenario results}

For the first scenario the varying characteristic of populations is probability $r=0,10^{-3}, 2 \cdot 10^{-3}, \ldots, 10^{-2}, 2$. $10^{-2}, \ldots, 10^{-1}, 1.5 \cdot 10^{-1}, \ldots, 1$ with 38 values. Besides the first scenario populations, we consider also the traditional PON populations (i.e., all RNs are passive), and allow for the IC with probability $r$. And so we consider $2 \cdot 38=76$ populations, and evaluate 7600 networks.

Fig. 5 shows the SA results in a logarithmic scale as a function of probability $r$. Each data point represents the sample mean. The line marked as " $r=0$ " shows the SA of 0.998921 for the first scenario with $r=0$, and the "baseline" shows the SA of 0.99897 for a traditional PON. Without the IC, i.e., $r=0$, the introduction of the active RNs decreases the SA, because the availability of an active $\mathrm{RN}$ is smaller than that of a passive RN.

However, even a small increase in $r$ causes a large increase in the SA for the first scenario, but a little increase for the traditional PON with the IC. For instance, when $r=10^{-2}$, i.e., when on average one ONU out of a hundred has the IC, the SA

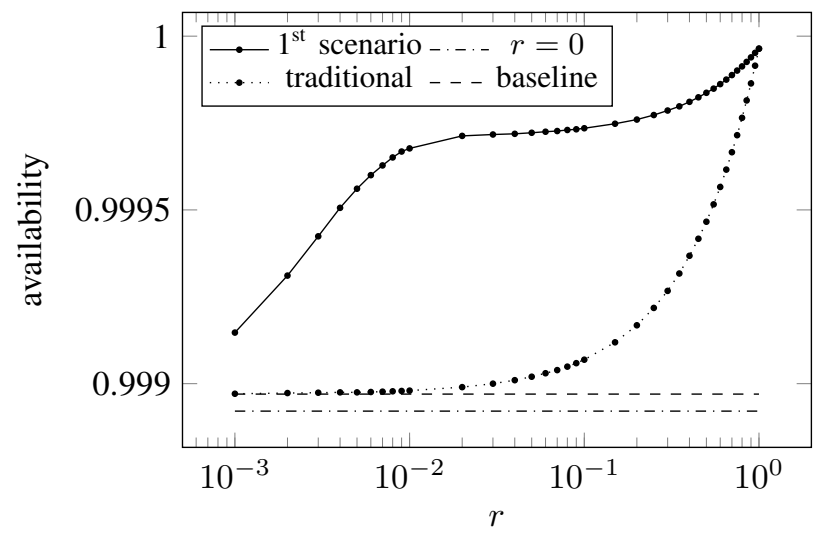

Fig. 5. Availability in the $1^{\text {st }}$ scenario as a function of $r$.

is 0.999677 , which corresponds to 2.83 hours of downtime per year, while the baseline SA of the traditional PON is 0.99897 , which corresponds to 9.02 hours of downtime per year. This is more than a threefold reduction in downtime.

For the first scenario networks, the SA rapidly increases as $r$ increases, because the presence of a single IC-ONU in a passive segment of the third stage allows all NIC-ONUs in that segment to reach the IC-ONU through an active $\mathrm{RN}$ in the second stage. This rapid trend continues up to the point when most passive segments in the third stage have an ICONU, and after that point adding more IC-ONUs does not increase rapidly the SA, as shown by the nearly-flat SA for $10^{-2}<r<10^{-1}$. For $r>10^{-1}$ the SA increases linearly because the high availability of the IC-ONUs increases directly the mean ONU SA.

In the evaluation we allowed the traditional PONs to be capable of the IC in order to show that the active RNs used in the first scenario help realize the full potential of the IC, which is evident for the small and practical values of $r$. Without the active RNs, the SA increases linearly as a function of $r$, as is the case for the traditional PONs with the IC.

\section{B. Second scenario results}

For the second scenario the probability $r$ and probability $q$ vary. Probability $r$ varies as in the first scenario, and $q=0,0.05,0.1, \ldots, 1$ has 21 values. Therefore there are $38 \cdot 21=798$ network populations considered, and 79800 networks evaluated.

The results are shown in Fig. 6 for $r \neq 0$ because of the logarithmic scale, and in Fig. 7 for $r=0$. A data point in the figures represents the sample mean of the SA. The baseline SA of 0.99897 of a traditional PON without the IC is shown in Fig. 6 as the gray plane, and in Fig. 7 as the dashed line.

For $q=0$, the SA is the same as for the traditional PONs in Fig. 5, because these networks do not have active RNs. The SA increases together with the increasing $r$ and $q$, but the increase is not as impressive as for the first scenario, since in the first scenario active RNs are positioned strategically in the second stage where they can interconnect a large number of 


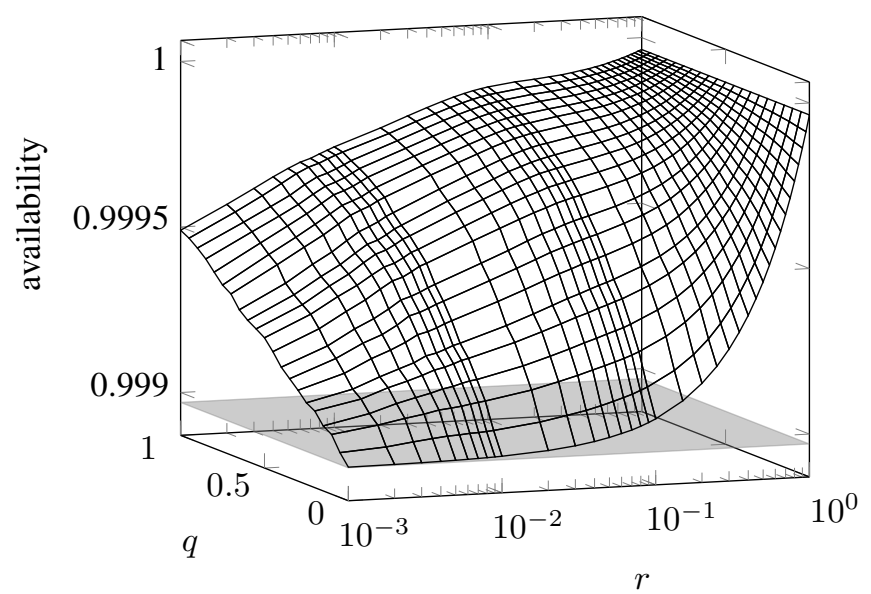

Fig. 6. Availability in the $2^{\text {nd }}$ scenario as a function of $r$ and $q$.

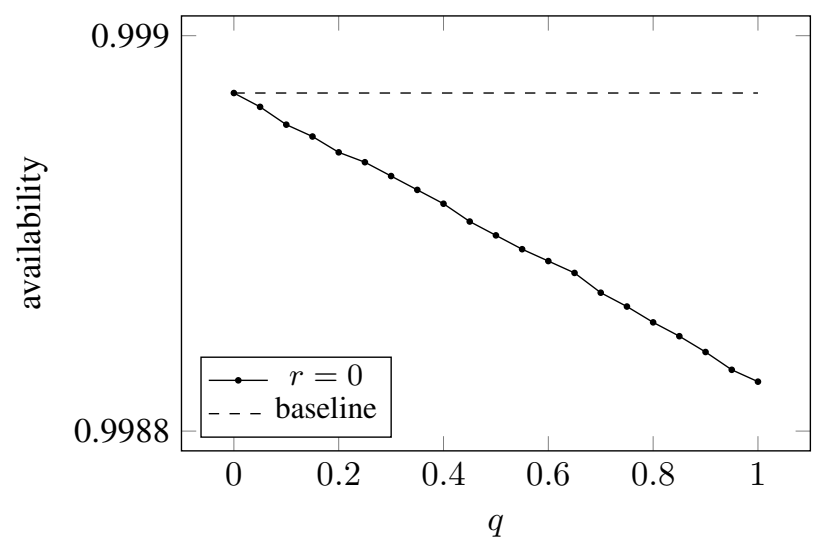

Fig. 7. Availability in the $2^{\text {nd }}$ scenario as a function of $q$ with $r=0$.

NIC-ONUs to a single IC-ONU, while in the second scenario an active RN can land in the third stage where it is less useful.

Fig. 7 shows that the SA decreases when active RNs are introduced, and when there are no IC-ONUs in the network, i.e., $r=0$. These results validate the $\mathrm{SA}$ calculation.

\section{CONCLUSION}

We proposed the novel idea of interoperator fixed-mobile network sharing, and showed that the service downtime can be reduced threefold by introducing a small number of active remote nodes and providing interoperator communication to as little as $1 \%$ of all optical network units. The interoperator communication can be delivered wirelessly between mobile base stations or with a fiber connecting optical network units.

The proposed solution would require the installation of a few active nodes and updating the software in network nodes, while the hardware of regular users would require no changes. The deployment of the active nodes could be rolled out in stages when needed, and in those areas where the resiliency is needed most, i.e., in a business district.

The proposed sharing allows for dynamic reconfiguration, since the interoperator communication can be carried out wirelessly by base stations. An operator can easily start or end the sharing with various operators, which would encourage competition.

Future work could concentrate on 1) generalizing the proposed sharing to other mobile backhaul types, like the microwave backhaul; 2) optimizing the placement of active remote nodes; 3 ) optimizing the selection of optical network units for interoperator communication; 4) optimizing various economic metrics, such as revenue, or the risk of liability due to service failure; 5) studying incentives which would foster sharing, and rules which would discourage cheating; 6) researching various aspects related to fixed-mobile networks, such as cognitive radio, various static and dynamic traffic models, cognitive radio, or coordinated multipoint transmission; and 7) generalizing the proposed sharing to any number of operators.

\section{ACKNOWLEDGMENTS}

This work was supported by the postdoctoral fellowship number DEC-2013/08/S/ST7/00576 from the Polish National Science Centre. The numerical results were obtained using PL-Grid, the Polish supercomputing infrastructure.

\section{REFERENCES}

[1] L. Doyle, J. Kibiłda, T. Forde, and L. DaSilva, "Spectrum without bounds, networks without borders," Proceedings of the IEEE, vol. 102, no. 3, pp. 351-365, March 2014.

[2] A. Khan, W. Kellerer, K. Kozu, and M. Yabusaki, "Network sharing in the next mobile network: TCO reduction, management flexibility, and operational independence," IEEE Communications Magazine, vol. 49, no. 10 , pp. 134-142, Oct 2011.

[3] C. Ranaweera, E. Wong, C. Lim, and A. Nirmalathas, "Next generation optical-wireless converged network architectures," IEEE Network, vol. 26, no. 2, pp. 22-27, March 2012.

[4] J.-i. Kani, F. Bourgart, A. Cui, A. Rafel, M. Campbell, R. Davey, and S. Rodrigues, "Next-generation PON-part I: technology roadmap and general requirements," IEEE Communications Magazine, vol. 47, no. 11, pp. 43-49, November 2009.

[5] L. Kazovsky, S.-W. Wong, T. Ayhan, K. Albeyoglu, M. R. N. Ribeiro, and A. Shastri, "Hybrid optical-wireless access networks," Proceedings of the IEEE, vol. 100, no. 5, pp. 1197-1225, May 2012.

[6] J. Beas, G. Castañon, I. Aldaya, A. Aragon-Zavala, and G. Campuzano, "Millimeter-wave frequency radio over fiber systems: a survey," IEEE Communications Surveys and Tutorials, vol. 15, no. 4, pp. 1593-1619, Fourth Quarter 2013.

[7] M. Ali, G. Ellinas, H. Erkan, A. Hadjiantonis, and R. Dorsinville, "On the vision of complete fixed-mobile convergence," Journal of Lightwave Technology, vol. 28, no. 16, pp. 2343-2357, 2010.

[8] D. Breuer, E. Weis, S. Gosselin, T. Mamouni, and J. Torrijos, "Unified access and aggregation network allowing fixed and mobile networks to converge," in Optical Fiber Communication Conference (OFC), 2013, March 2013, paper OTu2E.3.

[9] N. Ghazisaidi, M. Scheutzow, and M. Maier, "Survivability analysis of next-generation passive optical networks and fiber-wireless access networks," IEEE Transactions on Reliability, vol. 60, no. 2, pp. 479492, June 2011.

[10] H. Harada, Y. Alemseged, S. Filin, M. Riegel, M. Gundlach, O. Holland, B. Bochow, M. Ariyoshi, and L. Grande, "IEEE dynamic spectrum access networks standards committee," IEEE Communications Magazine, vol. 51, no. 3, pp. 104-111, March 2013.

[11] E. Jorswieck, L. Badia, T. Fahldieck, E. Karipidis, and J. Luo, "Spectrum sharing improves the network efficiency for cellular operators," IEEE Communications Magazine, vol. 52, no. 3, pp. 129-136, March 2014.

[12] C. Chan, M. Attygalle, and A. Nirmalathas, "Remote repeater-based EPON with MAC forwarding for long-reach and high-split-ratio passive optical networks," IEEE/OSA Journal of Optical Communications and Networking, vol. 2, no. 1, pp. 28-37, January 2010. 
[13] M. Mahloo, J. Chen, L. Wosinska, A. Dixit, B. Lannoo, D. Colle, and C. Machuca, "Toward reliable hybrid WDM/TDM passive optical networks," IEEE Communications Magazine, vol. 52, no. 2, pp. S14S23, February 2014.

[14] I. Szcześniak. (2015, January) The website of the AVAILA software. [Online]. Available: http://www.irkos.org/availa 